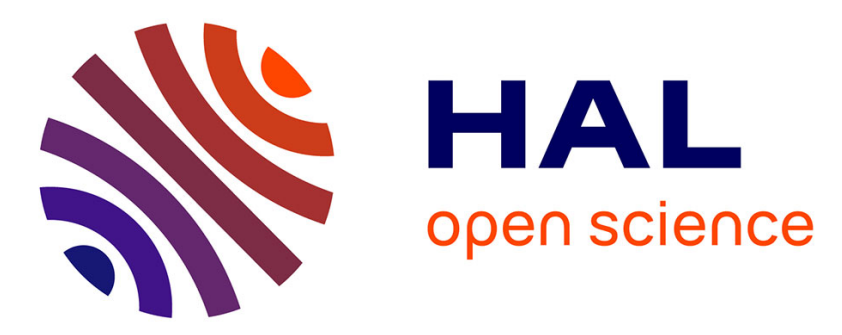

\title{
Innovative Multilayer OTS Selectors for Performance Tuning and Improved Reliability
}

C. Laguna, N. Castellani, M. Bernard, N. Rochat, D. Rouchon, C. Sabbione, J. Garrione, E. Nolot, G. Bourgeois, M.-C. Cyrille, et al.

\section{To cite this version:}

C. Laguna, N. Castellani, M. Bernard, N. Rochat, D. Rouchon, et al.. Innovative Multilayer OTS Selectors for Performance Tuning and Improved Reliability. IMW 2020 - 2020 IEEE International Memory Workshop, May 2020, Dresde, Germany. pp.1-4, 10.1109/IMW48823.2020.9108130 . cea02930899

\section{HAL Id: cea-02930899} https://hal-cea.archives-ouvertes.fr/cea-02930899

Submitted on 4 Sep 2020

HAL is a multi-disciplinary open access archive for the deposit and dissemination of scientific research documents, whether they are published or not. The documents may come from teaching and research institutions in France or abroad, or from public or private research centers.
L'archive ouverte pluridisciplinaire HAL, est destinée au dépôt et à la diffusion de documents scientifiques de niveau recherche, publiés ou non, émanant des établissements d'enseignement et de recherche français ou étrangers, des laboratoires publics ou privés. 


\title{
Innovative Multilayer OTS Selectors for Performance Tuning and Improved Reliability
}

\author{
C. Laguna ${ }^{1,2}$, N. Castellani ${ }^{1}$, M. Bernard ${ }^{1}$, N. Rochat ${ }^{1}$, D. Rouchon ${ }^{1}$, C. Sabbione ${ }^{1}$, \\ J. Garrione ${ }^{1}$, E. Nolot $^{1}$, G. Bourgeois ${ }^{1}$, M. C. Cyrille ${ }^{1}$, L. Militaru ${ }^{2}$, A. Souifi ${ }^{2}$, \\ G. Navarro ${ }^{1}$ and E. Nowak ${ }^{1}$ \\ ${ }^{1}$ CEA, LETI, MINATEC Campus, 17 rue des Martyrs, 38054 GRENOBLE Cedex 9, France. \\ ${ }^{2}$ Université de Lyon, INL-UMR CNRS 5270, INSA Lyon, 7 av. Jean Capelle, 69621 VILLEURBANNE, France.
}

\begin{abstract}
In this paper, we investigate an innovative Ovonic Threshold Switching Selector (OTS) based on Multilayer structure (ML). Thanks to physico-chemical analysis and electrical characterization we show how MLs properties and structure can be tuned thanks to the engineering of each individual layer stoichiometry, thickness and interfaces. Ge/N-doped SbSe-based MLs OTS are analyzed by FTIR and Raman spectroscopy revealing the structural features present in the as-deposited materials and the strong interaction among individual layers at interfaces. We demonstrate the improved variability control of electrical parameters wrt standard OTS achieved by co-sputtering technique, and the high endurance capability of MLs OTS up to more than $2 \cdot 10^{9}$ cycles with stable nA leakage current. Moreover, we show how Ge-N bonds play a huge role on OTS thermal stability at $400^{\circ} \mathrm{C}$ and how they can be tuned more easily in ML OTS. These developments pave the way towards a new class of OTS materials and their engineering, ensuring high temperature stability and best tuning of electrical performances.
\end{abstract}

\section{INTRODUCTION}

Ovonic Threshold Switching technology (OTS), based on amorphous chalcogenide materials, is considered among the most promising Back-End Selector because of its demonstrated reliability in terms of high ON/OFF selectivity, high endurance and high switching speed [1]. Recent developments of OTS materials showed the strong sensitivity of device electrical performance and electrical parameters variability on composition and doping [2]-[4]. This is at the expense of an increased material complexity, dealing with even more of five elements in the same alloy. Moreover, high temperature exposure could induce segregation and crystallization phenomena in amorphous chalcogenides, degrading the OTS switching behavior. We showed in a previous work [5] the possibility to combine reliable switching properties and low leakage current introducing a low bandgap As-Te alloy in a high bandgap Se-rich Ge-Se alloy, achieving tunable OTS performances with a relative reduced material complexity, because of the pseudo-binary nature of the fabricated alloy.

In this work, we take advantage of the switching properties of a chalcogenide alloy based on Sb-Se (SS) stacked with Ge layers and selectively doped with $\mathrm{N}$. Thanks to the comparison

We acknowledge the support of European commission, French State and Auvergne-Rhône Alpes region through the funding of ECSEL project WAKEMEUP part of IPCEI (Important project of commun european interest) microelectronics and French Nano2022 program.

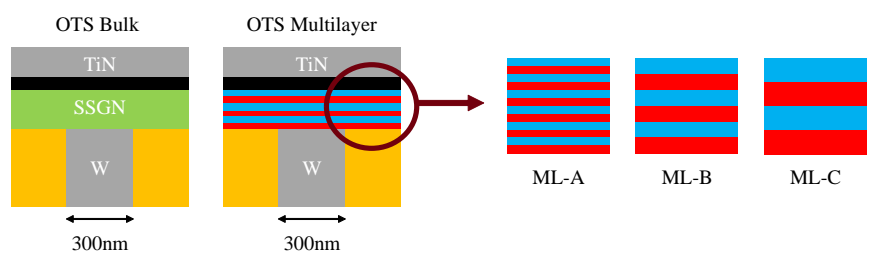

Fig. 1. Simplified description of our OTS ML device compared to standard OTS. The bottom electrode consists in a tungsten plug with a diameter of $\sim 300 \mathrm{~nm}$. A carbon intermediate electrode is fabricated between the top electrode and the OTS material to prevent intermixing among the two during cycling operations.

of electrical parameters of OTS multilayer structure (ML) with a standard OTS based on a bulk layer $(\mathrm{Ge} / \mathrm{N}$-doped SS or SSGN, Fig. 1) designed considering our previous studies on Ge-Se-Sb-N (GSSN) [4], we study the switching performance, the capability to ensure material thermal stability and the endurance in these innovative selectors. Moreover, by physico-chemical analysis we show how ML structure does not depend only on each single layer structure but also on interfaces interactions, leading to a proper control of material properties. This result leads to a lower variability of OTS electrical parameters, shown even after a Back-EndOf-Line-like thermal stress protocol (BEOL). We demonstrate that through an innovative OTS ML based on two different relatively simple alloys, we can target the material stability at high temperatures selectively doping the layers, and tuning the selector switching performances, by properly engineering interfaces, thickness and individual layer stoichiometry.

\section{Material Characterization of OTS MULTILAYERS}

We fabricated OTS MLs by alternative magnetron reactive sputtering from SS and Ge targets doping selectively with N. Three materials were realized with invidual layer thicknesses ranging from $1 \mathrm{~nm}$ to $\sim 5 \mathrm{~nm}$. The total thickness of the stack is kept constant, therefore the total number of interfaces is higher for thinner layers. We will refer in the following to theses material as ML-A, ML-B and ML-C respectively. SSGN layer was deposited by standard magnetron co-sputtering deposition technique.

We characterize materials using IR spectroscopy. Fig. 2 shows FTIR spectra of SSGN and MLs as deposited and after a 


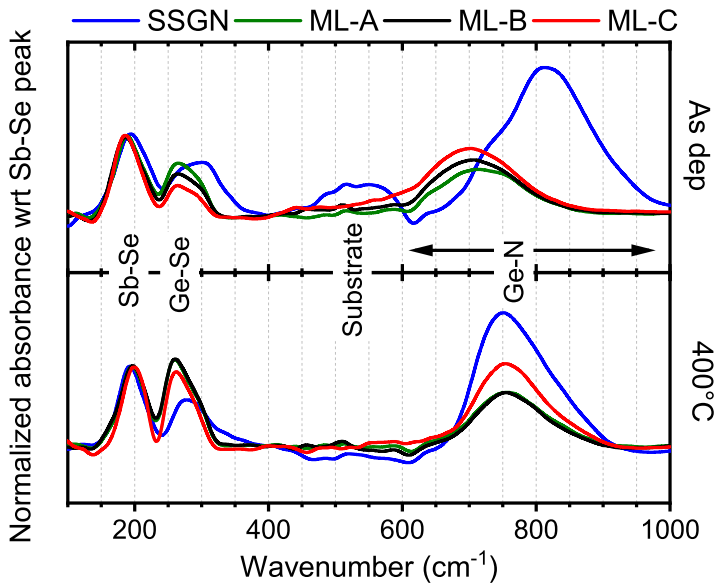

Fig. 2. FTIR spectra of SSGN and MLs (top) as deposited and (bottom) after a $400^{\circ} \mathrm{C}$ annealing. The peak at $266 \mathrm{~cm}^{-1}$ corresponds to $\mathrm{GeSe}_{4}\left(v_{3}\right.$ mode) which is priviledged in multilayer structure while the shoulder at $300 \mathrm{~cm}^{-1}$ should correspond to GeSeGe ( $v_{1}$ mode) [6], [7] that is more intense in bulk SSGN. After annealing, SSGN bond network evolves to a structure comparable to MLs.

monitored temperature ramp up of $10^{\circ} \mathrm{C} / \mathrm{min}$ stopped at $400^{\circ} \mathrm{C}$ for $\sim 1$ min. All the spectra are normalized wrt Sb-Se peak as reference. We observe the presence in all the layers of expected $\mathrm{Sb}-\mathrm{Se}$ and $\mathrm{Ge}-\mathrm{N}$ bonds. The presence of Ge-Se bonds even in MLs certifies the strong inter-layers interaction and the high probability of $\mathrm{Ge}-\mathrm{Se}$ formation [7]. Ge-Se bonds are important to preserve the amorphous layer thermal stability and to avoid the OTS mechanism degradation (i.e. layer crystallization). The interfaces in our MLs play the role of controlling such feature, together with SS layer stoichiometry and $\mathrm{N}$ doping control. In Fig. 3 this phenomenon is evidenced by the increasing of Ge-Se wrt Sb-Se and Ge-N moving toward a higher interface number in ML-A, even boosted after annealing at $400^{\circ} \mathrm{C}$. Ge-N features in bulk SSGN, important for glass stability, evolves toward a more defined GeN peak [8] that is compatible with the one already present in as-dep MLs, in which it is stable and only slightly increasing after annealing at a higher wavenumber presented in Fig. 2. The higher the content of $\mathrm{GeN}$ in the layers, the higher the final thermal stability expected. Selective doping reacheses a higher content of $\mathrm{N}$ in SSGN, which explains the higher intensity of Ge-N peak.

Fig. 4 shows Raman spectra for SSGN and MLs as-deposited and after annealing. The major band around $200 \mathrm{~cm}^{-1}$ is the convolution of Ge-Se and Sb-Se that is present in both bulk and ML structures. The band near $160 \mathrm{~cm}^{-1}$ can be assigned to vibration mode of a $\mathrm{Sb}$-Sb. In the case of MLs this mode remains stable even after annealing, that could be linked to the structure of SS involving $\mathrm{Se}_{2} \mathrm{Sb}_{-} \mathrm{SbSe}_{2}$ features [10], [11]. The presence of amorphous Ge layers, and the unchanged MLs associated spectra after annealing, support the integrity of the MLs under thermal stress.

The inter-layers interaction, the stoichioemtry and the selective $N$ doping in Ge+Sb-Se-based OTS MLs lead to a higher control of the material structure and of its thermal stability

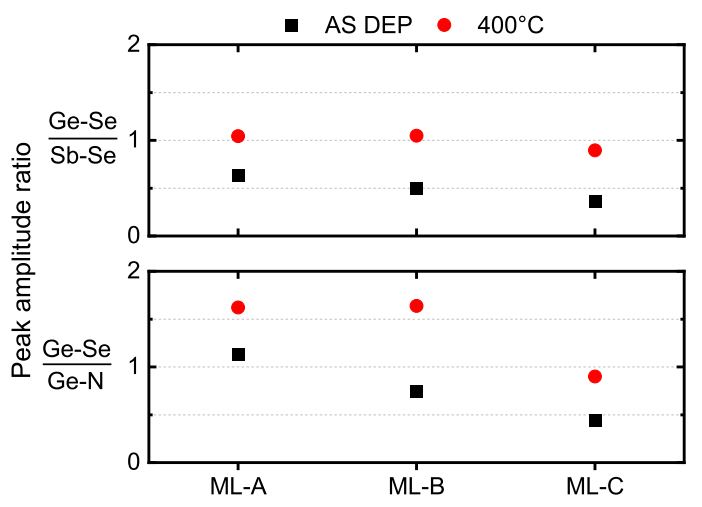

Fig. 3. Intensity ratios of $\mathrm{Ge}-\mathrm{Se}$ peaks wrt $\mathrm{Sb}-\mathrm{Se}$ (top) and wrt $\mathrm{Ge}-\mathrm{N}$ peaks (bottom) for ML-A, ML-B and ML-B as deposited and after a $400^{\circ} \mathrm{C}$ annealing, extracted from Fig. 2

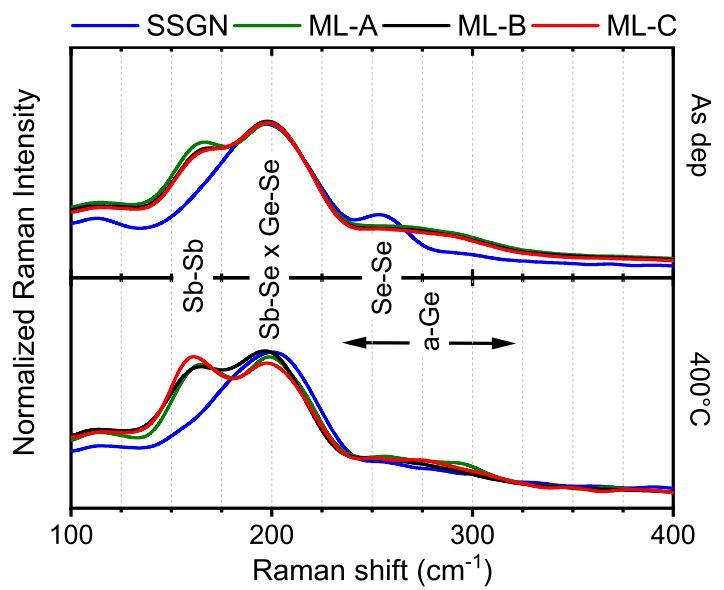

Fig. 4. Raman spectra for SSGN and MLs as deposited (top) and and after $400^{\circ} \mathrm{C}$ annealing (bottom). All spectra are normalized using the main feature at $200 \mathrm{~cm}^{-1}$ that results from correlation of [ $\left.\mathrm{SbSe}_{3 / 2}\right]$ and $\left[\mathrm{GeSe}_{4 / 2}\right]$ features. Homopolar $\mathrm{Sb}-\mathrm{Sb}$ bonds are part of the more complex feature $\left(\mathrm{Se}_{2}\right) \mathrm{Sb}(\mathrm{Ge})-\mathrm{Sb}\left(\mathrm{Se}_{2}\right)$ [10], [11] that stabilizes after annealing.

wrt standard bulk SSGN.

\section{ELECTRICAL CHARACTERIZATION OF OTS MUlTILAYERS SELECTORS}

We performed the electrical characterization of OTS Multilayers analytical selector devices, deposited on a $300 \mathrm{~nm} \mathrm{~W}$ plug. Devices were tested in both quasi-static (DC) and pulsed (AC) mode.

Fig. 5 presents the post-firing DC current-vs-voltage (IV) curves of SSGN and ML-A as an example of the high compatibility of switching behavior between bulk and ML. Leakage current $\left(\mathrm{I}_{\text {off }}\right)$ is defined for each material as the current measured at half of its switching voltage $\left(\mathrm{V}_{\mathrm{th}} / 2\right)$ and $\mathrm{I}_{\mathrm{h}}$ is the holding current.

All the experiments were performed on populations of 20 devices. In Fig. 6 we report the subthreshold IV curves before and after firing (DC mode), in order to compare the conduction mechanism in SSGN wrt MLs. Theses curves can be fitted using Poole-Frenkel conduction relation:

$$
\ln (I / V) \propto \beta_{P F} \cdot \sqrt{V}
$$




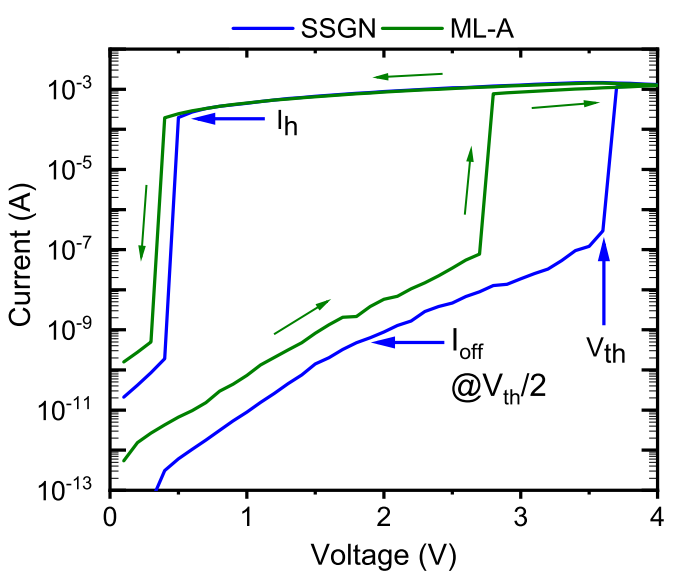

Fig. 5. Quasi-static curves for SSGN and ML-A, demonstrating the compatibility of the switching behavior between OTS ML and bulk OTS. We define the leakage current $\left(\mathrm{I}_{\mathrm{off}}\right)$, measured at $\mathrm{V}_{\mathrm{th}} / 2$, and the holding current $\left(\mathrm{I}_{\mathrm{h}}\right)$.

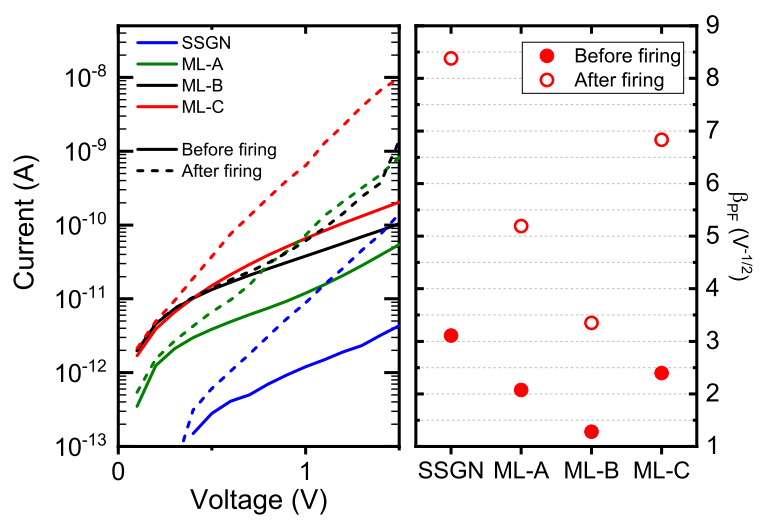

Fig. 6. Subthreshold IV characteristics before and after firing for SSGN and MLs (only median curves are shown) (left). Subthreshold conductivity slope $\beta_{P F}$ is calculated for all the samples (right).

where $\beta_{P F}$ parameter reveals the subthreshold dependency of the layers conductivity, and it is calculated before and after firing. Firing operation induces a material evolution, that appears similar in all the samples. The increase of subthreshold non-linearity is higher in bulk layer wrt MLs samples (i.e. higher $\beta_{P F}$ ) that we think could be correlated with the higher material disorder after firing operation, already observed in the variability of electrical parameters.

In Fig. 7 and Fig. 8 we report all the electrical parameters measured in $\mathrm{AC}$ for the materials investigated, after $10^{4}$ and $10^{8}$ cycles. SS shows OTS behavior up to $10^{2}$ cycles, with higher dispersion of starting fire voltage $\left(\mathrm{V}_{\text {fire }}\right)$ and following $\mathrm{V}_{\mathrm{th}}$, before to trigger crystallization and loss of the OTS behavior. SSGN presents both higher $\mathrm{V}_{\text {fire }}$ and $\mathrm{V}_{\text {th }}$ and higher variability from starting cycles wrt MLs. Lower $\mathrm{V}_{\text {th }}$ variability in OTS has a strong importance for the reliability of reading operation once co-integrated with resistive memory devices [12]. We think that the gradual reduction of the $V_{\text {fire }}$ from bulk to MLs can be linked to SS layers thickness. Indeed SS has a lower resistivity [13] compared to N-doped Ge layer and a lower firing voltage wrt to SSGN. Therefore, firing operation

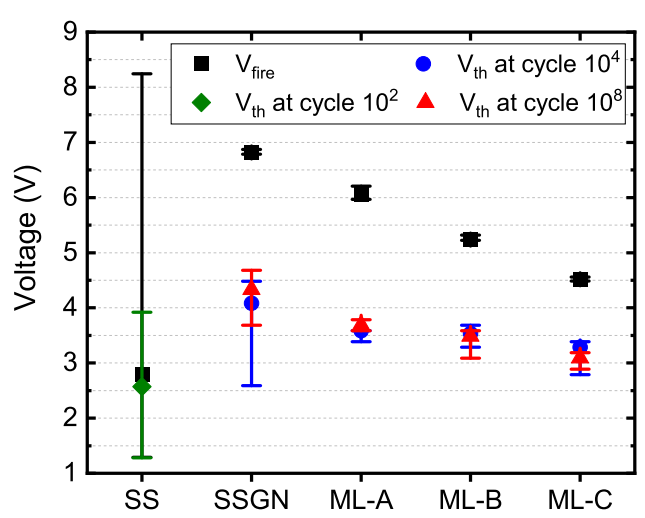

Fig. 7. $\mathrm{V}_{\text {fire }}$ and $\mathrm{V}_{\text {th }}$ after $10^{4}$ and $10^{8}$ cycles for SSGN and MLs. SSGN shows higher $V_{\text {fire }}$ and $V_{\text {th }}$ values and higher variability wrt MLs. SS parameters are shown only up to $10^{2}$ cycles since after the material triggers crystallization.

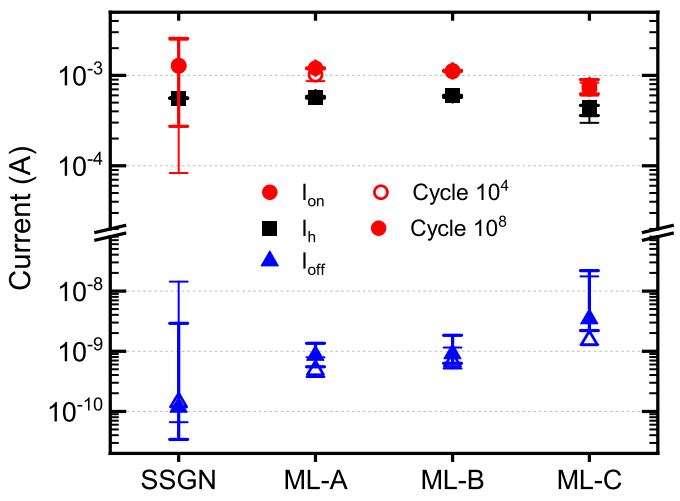

Fig. 8. Electrical parameters $I_{o n}, I_{h}$ and $I_{\text {off }}$ after $10^{4}$ and $10^{8}$ cycles for SSGN and MLs. Identical results are achieved with both RESET and SET-like pulses conventionally used for Phase-Change Memory applications (not reported for graph clarity).

becomes easier and achieved at lower voltage when SS layer thickness increases.

SSGN shows lower leakage current compared to MLs (Fig. 7), but it confirms the higher variability of both ON (e.g. ON current $\mathrm{I}_{\mathrm{on}}$ ) and OFF parameters even after $10^{8}$ cycles. An $\mathrm{I}_{\text {on }} / \mathrm{I}_{\text {off }}$ ratio of more than $10^{6}$ is ensured in thinnest MLs with $\mathrm{I}_{\mathrm{off}}$ in $\mathrm{nA}$ range with a reduced dispersion.

Endurance capability of OTS ML solution is demonstrated in Fig. 9 where the endurance of ML-A reaches more than $2 \cdot 10^{9}$ cycles with extremely low cycle-to-cycle variation of $\mathrm{I}_{\text {off }}$ and $\mathrm{I}_{\mathrm{on}}$ parameters.

In order to evaluate the thermal stability of MLs devices, we annealed the devices with a thermal budget of $30 \mathrm{~min}$ at $400^{\circ} \mathrm{C}$. Fig. 10 reports the value of the starting resistance of the devices $\left(\mathrm{R}_{\mathrm{virgin}}\right)$, before and after annealing. While ML-A and ML-B have crystallized, SSGN and ML-C, even if we observe a starting of crystallization, show a totally compatible behavior among selector requirements. The trend is in line with material analyses, where the GeN presence, likely responsible for the glass stability, is found higher in SSGN (Fig. 2). ML-C, 


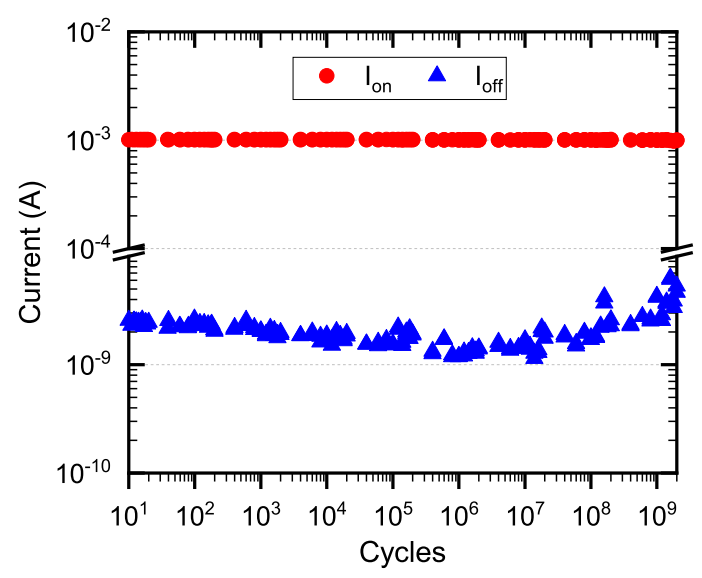

Fig. 9. $\mathrm{I}_{\text {off }}$ and $\mathrm{I}_{\mathrm{on}}$ along cycling up to $2 \cdot 10^{9}$ cycles for ML-A.

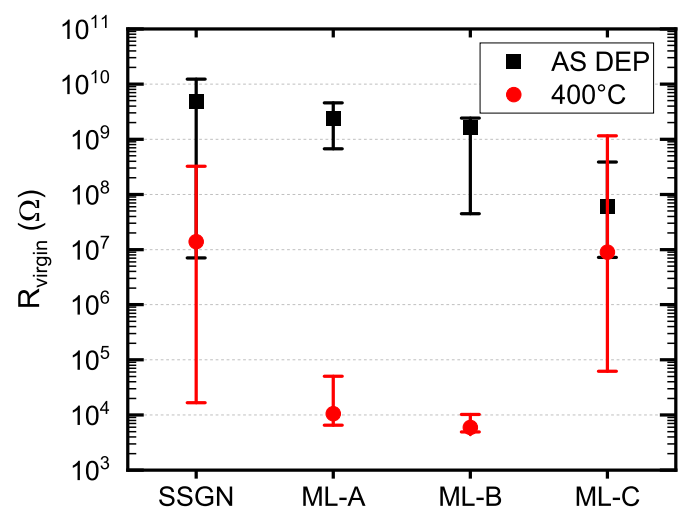

Fig. 10. $\mathrm{R}_{\text {virgin }}$ measured at $\mathrm{V}_{\text {th }} / 2$ before and after extreme thermal budget of $30 \mathrm{~min}$ at $400^{\circ} \mathrm{C}$.

even though its $\mathrm{N}$ doping rate is three times inferior to SSGN, shows comparable thermal stability. This result highlights the capability of ML solution to improve the structure control of the layer, in particular enabling selective bonds formation.

Focusing on SSGN and ML-C, Fig. 11 shows their IV curves before and after firing after annealing. The annealing increases significantly the subthreshold conductivity in SSGN wrt ML-C. Moreover, the electric field dependency of subthreshold conductivity remains lower in ML-C (i.e. lower $\left.\beta_{P F}\right)$, confirming a likely lower disorder even after annealing.

Finally, in Fig. 12 we report the post annealing spread in \% of $\mathrm{V}_{\text {th }}$ and $\mathrm{I}_{\text {off }}$ measured after $10^{4}$ and $10^{8}$ cycles for SSGN and ML-C. Variability of electrical parameters is always lower for ML-C wrt SSGN, even after cycling.

OTS MLs show stable nA range leakage current and high endurance capability up to more than $2 \cdot 10^{9}$ cycles featuring a huge reduction of electrical parameters variability. Thermal stability at $400^{\circ} \mathrm{C}$ is in line with the one of bulk reference layer, showing the capability of $M L$ solution to improve the structure control by selective bonds formation.

\section{CONCLUSIONS}

We investigated innovative OTS ML selectors based on $\mathrm{N}$-doped $\mathrm{Ge}$ and $\mathrm{Sb}-\mathrm{Se}$. We showed how the inter-layers

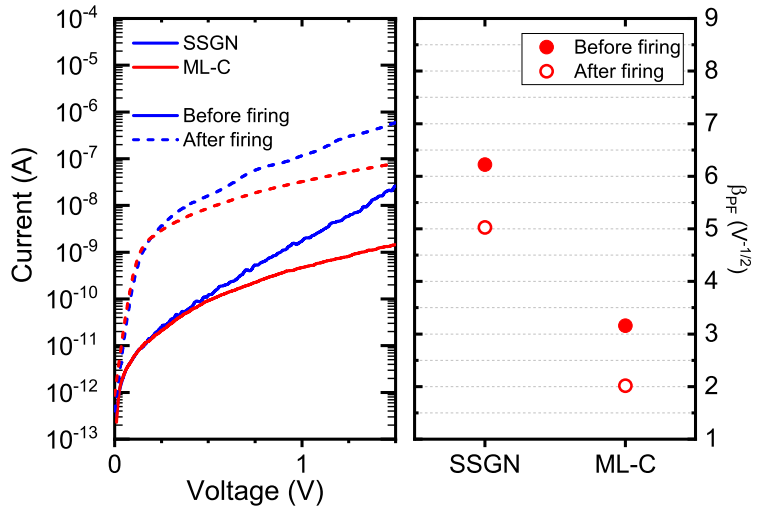

Fig. 11. Subthreshold IV curves before and after firing for SSGN and ML-C after the $400^{\circ} \mathrm{C} / 30 \mathrm{~min}$ annealing (only median curves are shown) (left) and correspondent subthreshold conductivity slope $\beta_{P F}$ (right).

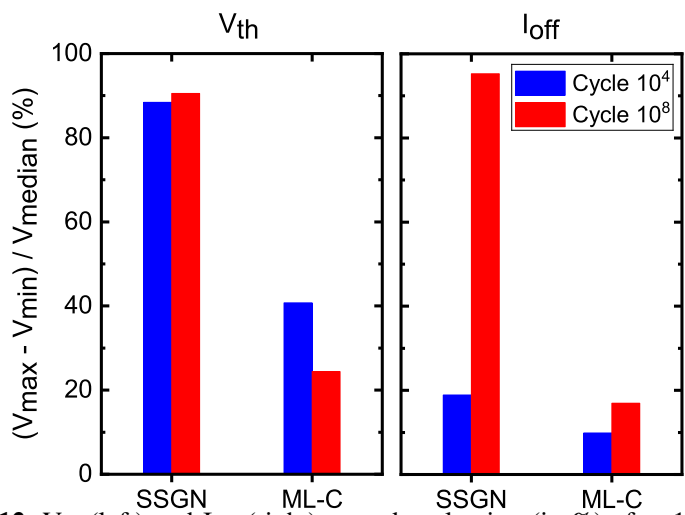

Fig. 12. $\mathrm{V}_{\text {th }}$ (left) and $\mathrm{I}_{\text {off }}$ (right) spread evaluation (in $\%$ ) after $10^{4}$ and $10^{8}$ cycles for SSGN and ML-C after $400^{\circ} \mathrm{C} / 30 \mathrm{~min}$ annealing.

interaction, demonstrated by FTIR and Raman analyses, the stoichioemtry and the selective $\mathrm{N}$ doping in our OTS MLs lead to a higher control of the material structure and of its thermal stability. We presented the improved variability control of electrical parameters and high endurance capacity up to $2 \cdot 10^{9}$ cycles of MLs OTS. Finally, we show how Ge-N bonds play a huge role on OTS thermal stability at $400^{\circ} \mathrm{C}$ and how they can be tuned more easily in ML OTS. We think that these results open to a new class of OTS devices and to their engineering, ensuring high temperature stability and best tuning of electrical performances.

\section{REFERENCES}

[1] G. W. Burr et al., J. Vac. Sci. Technol. B, vol. 32(4), pp. 040802, 2014.

[2] H. Y. Cheng et al., in Proc. 2017 IEDM, pp. 2.2.1-2.2.4, 2017.

[3] D. Garbin et al., in Proc. 2019 IEDM, 35.1.1-35.1.4,2019.

[4] A. Verdy et al., in Proc. 2018 IEDM, 37.4.1-37.4.4, 2018.

[5] A. Verdy et al., in Proc. IMW 2019,2-4, pp.1-4, 2019.

[6] P. Sharma et al., J. Alloys Compd., vol. 480, pp. 934-937, 2009.

[7] D.R. Goyal et al., J. Non-Cryst. Solids, vol. 183, pp. 182-185, 1995.

[8] A. Verdy et al., in Proc. 2018 IMW, 10.1109, pp. 1-4, 2018.

[9] M. Olivier et al., Opt. Express, vol. 4(3), pp. 525-540, 2014.

[10] E. Baudet et al., J. Non-Cryst. Solids, vol. 44, pp.64-72, 2016.

[11] D. Kau et al, in Proc. 2009 IEDM, 27.1.1-27.1.4, 2009.

[12] F.A. El-Salam et al., Vacuum, vol. 44, pp. 1009-1013, 1993. 